\title{
Analysis of Heart Rate Variability in the Presence of Ectopic Beats Using the Heart Timing Signal
}

\author{
Javier Mateo* and Pablo Laguna, Member, IEEE
}

\begin{abstract}
The time-domain signals representing the heart rate variability (HRV) in the presence of an ectopic beat exhibit a sharp transient at the position of the ectopic beat, which corrupts the signal, particularly the power spectral density (PSD) of the HRV. Consequently, there is a need for correction of this type of beat prior to any HRV analysis. This paper deals with the PSD estimation of the HRV by means of the heart timing (HT) signal when ectopic beats are present. These beat occurrence times are modeled from a generalized, continuous time integral pulse frequency modulation model and, from this point of view, a specific method for minimizing the effect of the presence of ectopic beats is presented to work together with the HT signal. By using both, a white noise driven autoregressive model of the HRV signal with artificially introduced ectopic beats and actual heart rate series including ectopic beats, the more usual methods of HRV spectral estimation are compared. Results of the PSD estimation error function of the number of ectopic beats are presented. These results demonstrate that the proposed method has one order of magnitude lower error than usual ectopic beats removal strategies in preserving PSD, thus, this strategy better recovers the original clinical indexes of interest.
\end{abstract}

Index Terms-Ectopic beat, heart rate variability, heart timing signal, integral pulse frequency modulation (IPFM) model, spectral analysis.

\section{INTRODUCTION}

$\mathbf{H}$ EART RATE variability (HRV) analysis is based on the study of the sinoatrial (SA) node activity as the source of repetitive impulses that generate normal heart beats. The normal activity of the SA node is assumed to be regulated, amongst others, by the sympathetic and parasympathetic neural systems. In addition to the SA node, other latent pacemakers exist throughout the heart. Normally, regular conduction of the electrical impulse from the SA node and the refractory period of the cells reject any other electrical source except those coming from the SA node. However, some of the additional pacemakers may, in certain cases, interpose additional electrical impulses that generate ectopic beats, which are usually manifested as a

Manuscript received January 24, 2002; revised October 18, 2002. This work was supported in part by the Comisión Interministerial de Ciencia y Tecnología (CICYT) and FEDER under Grant TIC2001-2167-C02-02, in part by the Consejo Superior de Investigación y Desarrollo (CONSI+D), and in part by Diputación General de Aragón (DGA) under Grant P075/2001. Asterisk indicates corresponding author.

* J. Mateo is with the Grupo de Tecnologías de las Comunicaciones (GTC), Instituto de Investigación en Ingeniería de Aragón (I3A), Universidad de Zaragoza, C/. María de Luna 1, 50018 Zaragoza, Spain (e-mail: jmateo@posta.unizar.es)

P. Laguna is with the Grupo de Tecnologías de las Comunicaciones (GTC), Instituto de Investigación en Ingeniería de Aragón (I3A), Universidad de Zaragoza, 50018 Zaragoza, Spain

Digital Object Identifier 10.1109/TBME.2003.808831 premature beat followed by a longer than normal heart period interval (RR) up to the next normal beat due to a compensatory delay [1, pp. 75-85]. In addition to ectopic beats, QRS complex misdetections can generate a similar effect to that of ectopic beats in HRV analysis. The time-domain signals associated with HRV exhibit a sharp transient at the ectopic beat, making it unusable, particularly in the power spectral density (PSD) estimate of HRV. An isolated ectopic beat can corrupt the PSD estimate because the broad-band frequency content of the impulse-like artifact, and in particular, frequency-domain indexes will be erroneously overestimated [2]-[5].

Different ectopic beat locations lead to different behavior. The ectopic focus location sometimes does not reset the normal activity of the SA node. The result is a missed normal beat, inhibited by the ectopic beat, followed by a normal beat placed at the same position as if the ectopic beat had not occurred [6, pp. 43-47]. This is usually the case when the ectopic focus location is ventricular and there is a cancellation of the electrical wavefronts of the ectopic beat and the regular beat expected to appear. Consequently, when the ectopic beat is rejected, the period between the two adjacent normal beats is approximately twice the mean heart period. In this case, reconstruction of the missed beat by intermediate insertion offers an approach that results in no substantial disturbance of the final PSD estimate [7]. Unfortunately, when the location of the ectopic focus is supraventricular, its electrical activity is able to reset the SA node activity resulting in a shortened period between the two adjacent normal beats, this period being noticeably smaller than twice the mean heart period. The simplest alternatives of including an intermediate beat or shifting the remaining beats will not produce valid solutions because both procedures substantially distort the PSD estimation of the HRV.

Recently, the heart timing (HT) signal was proposed [8] and it was shown that a better estimate of the HRV PSD can be obtained by using this signal in ectopic-beats-free situations. The heart timing signal becomes very sensitive to ectopic beats, and standard ectopic beat removal strategies, like removal/interpolation, are not sufficient for attenuation of the ectopic effect. In this paper, a complete procedure to analyze the HRV with the HT signal in real situations is presented. First, a method is proposed for the detection of irregular sinus beats (due to ectopic beats or QRS complex misdetections) that can affect the later PSD estimate of the HRV. Then, a solution is presented to minimize the effect of these types of anomalies in the series of beats and the corresponding PSD-derived clinical indexes. The proposed solution to be used together with HT signal, valid both if the ectopic beat has reset the activity of the SA node and if it has not been affected, is based on a generalization of the integral 
pulse frequency modulation (IPFM) model able to deal with the presence of ectopic beats. The proposed solution will be compared with other methods of estimating the PSD in the presence of ectopic beats in HRV signals modeled by means of an AR model with similar spectral distributions to those of real cases, but with simulations of the presence of a variable number of ectopic beats at different locations. The results will show the error in PSD estimation at the clinically defined frequency bands as function of the ectopic beat rate. These simulation results will be also corroborated by actual HRV signal analysis.

\section{METHODS}

\section{A. Identification of Anomalies on Beat Sequences}

The first stage in HRV studies is to obtain the time when each beat occurs. This process is achieved by detecting the QRS complexes from the ECG. In an automatic detection two kinds of anomalies can be found: Anomalies due to detector errors and anomalies due to ectopic beats. The detector errors can be false positive (FP) when a false beat is detected due to noise or a high amplitude $\mathrm{T}$ wave or false negative $(\mathrm{FN})$ when a real beat is missed due to a low amplitude QRS or noise masking. The anomalies caused by ectopic beats depend on the localization of the ectopic focus and are usually divided in supraventricular, if the focus is in the atria or the AV node, or ventricular if the focus is localized in the ventricle. Usually, the ventricular ectopic beat has a different morphology than a normal beat and the ectopic beat does not reset the normal activity of SA node. A normal beat is simply substituted by the ventricular ectopic beat. However, if the ectopic beat is supraventricular, its morphology is similar to that of a normal beat, but the SA node activity is reset, giving an interval between the adjacent normal beats that is appreciably lesser than a double normal-interval.

Given that these anomalies are frequent and notably affect the PSD estimation of the HRV, an algorithm for the detection of anomalies is proposed. In [9]-[11], different criteria for the rejection of anomalous intervals are set out. These criteria are based on beat location differences as heart period (HP) or heart rate (HR). Here, the proposed method is oriented to the beat location (actual or missed), not to the interval. To do so, it is necessary to identify the beat that causes the anomaly.

The criterion that has been established to detect the anomalous beat position is based on the fact that the variation of the instantaneous heart rate $(r(t))$ due to the normal beats is band limited since the SA node modulation is also band limited according to the IPFM model. So, it is possible to impose a threshold $(U)$ on the derivative of the instantaneous heart rate $\left(r^{\prime}(t)\right)$ to enable the acceptance of the beat location as belonging to a SA beat. It can be seen as the maximum allowed acceleration or deceleration of the heart rate due to normal beats. To estimate the derivative of the instantaneous heart rate at the location of the kth beat, $t_{k},\left(\widehat{r_{k}^{\prime}}=\widehat{r^{\prime}}\left(t_{k}\right)\right)$ the beat occurrence times, $t_{k \pm 1}$, of adjacent beats are used from the point of view of the IPFM model (see Section II-B-1) and Lagrange's interpolation formula. This condition can be written as (see the Appendix for derivation details)

$$
\left|\widehat{r_{k}^{\prime}}\right|=2\left|\frac{t_{k-1}-2 t_{k}+t_{k+1}}{\left(t_{k-1}-t_{k}\right)\left(t_{k-1}-t_{k+1}\right)\left(t_{k}-t_{k+1}\right)}\right|<U .
$$

When the condition in (1) is not met for some time instant $t_{k}$, some position $t_{k-1}, t_{k}$, or $t_{k+1}$ are anomalous. However, if $t_{k-1}$ was incorrect, the condition (1) would not be met in the previous step. So, $t_{k}, t_{k+1}$, or both are the candidates. To determine which one is the anomalous position, the condition (1) is tested in six different situations: by removing $t_{k}$, removing $t_{k+1}$, inserting an intermediate extra beat between $t_{k-1}$ and $t_{k}$, inserting a intermediate beat between $t_{k}$ and $t_{k+1}$, moving $t_{k}$ to the intermediate position between $t_{k-1}$ and $t_{k+1}$, and moving $t_{k+1}$ to the intermediate position between $t_{k}$ and $t_{k+2}$. If the criterion is now satisfied when removing, it implies a FP at the removal position; if the criterion is satisfied on insertion, this implies a FN and if satisfied when moving it typically implies an ectopic beat. If the condition is not met at any of the six situations, it is extended by considering possible consecutive FP, FN, or ectopic beats (two consecutive removals, insertions, or movements) and so on until the condition is satisfied by involving one more beat in each step. The criterion is to solve the anomaly by modifying the minor number of original beats. This method can detect consecutive FPs by sequentially deleting several beat positions, consecutive FNs by inserting evenly spaced beats and consecutive bad positioned (ectopic) beats by moving these to evenly spaced positions. The deleted, moved, or inserted beats are annotated consequently to avoid their detection as anomalous in further steps, and so that they will not be used as normal beats in the following processing stages. This serves to classify the anomaly and decide what the beat location $t_{k}$ is that does not come from SA activity.

Fig. 1 shows different kinds of anomalies found in the study of the ESDB database [12]. Each figure shows the ECG in the upper panel. In the lower panel, the original heart rate and the modified one are shown indicating which action has been carried out. Note that the new beat locations are not the definitive locations that will be introduced in HRV analysis, there are just gross positioning that classify the anomaly.

\section{B. Correction of Ectopic Beat Presence Effect}

Once the anomalous beat is identified, it is necessary to correct this effect in the studies of HRV. When HT signal is straightforward evaluated by $h t\left(t_{k}\right)=k T-t_{k}$ [8], after rejecting the ectopic beats and interpolating into the gaps, the estimated mean heart period, $T$, will be also affected by the ectopic gap and the HT values in the neighborhood of an ectopic beat will be largely mistaken due to the regular concordance lost between the integer $k$ and the beat position $t_{k}$. Because of this problem, we develop a method that works from the point of view of the IPFM model, simply by a generalization of the integer variable $k$ to a real variable $x$ as it will be shown.

1) IPFM Model in the Presence of Ectopic Beats: Many authors have assumed that the model IPFM explains the mechanism of the autonomic nervous system in controlling the heart rate. Different studies based on the IPFM model are presented in [13]-[19]. The IPFM model presupposes a modulating signal, which generates the beat occurrence times when acting through the model.

In [8], a continuous time generalization of the IPFM model was presented to support that the HT signal is a better alternative to the analysis of the HRV with respect to the conventional 

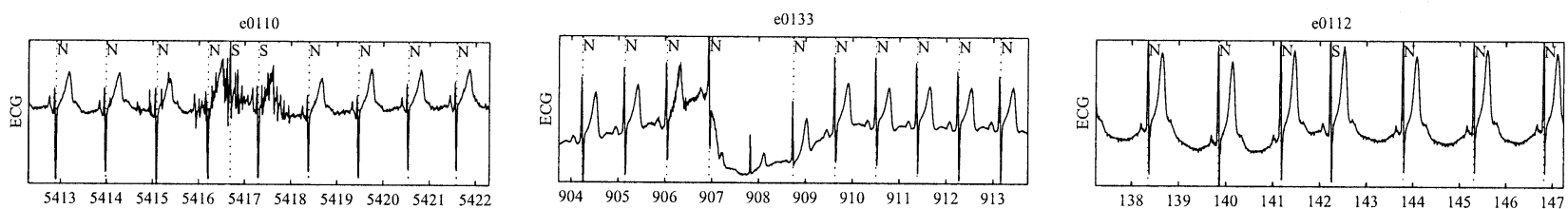

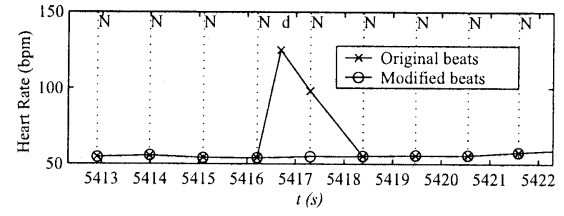

(a)
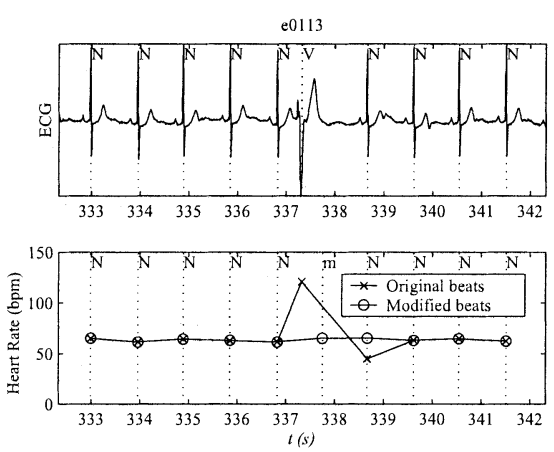

(d)

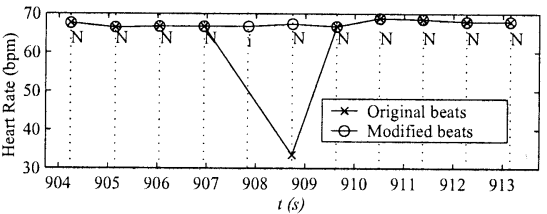

(b)
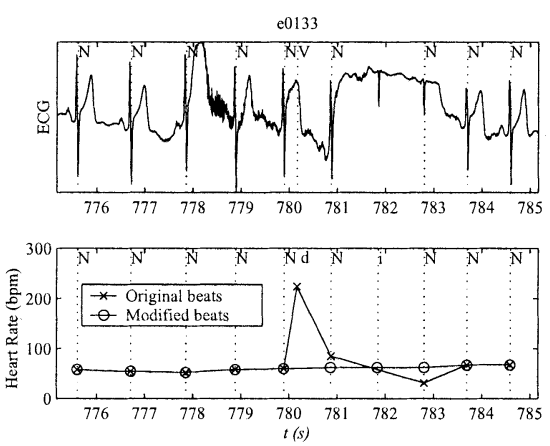

(e)

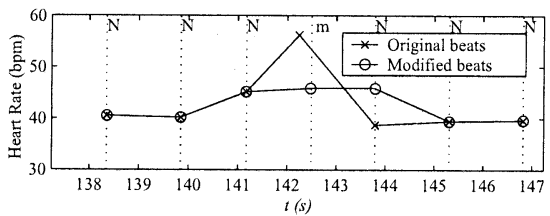

(c)
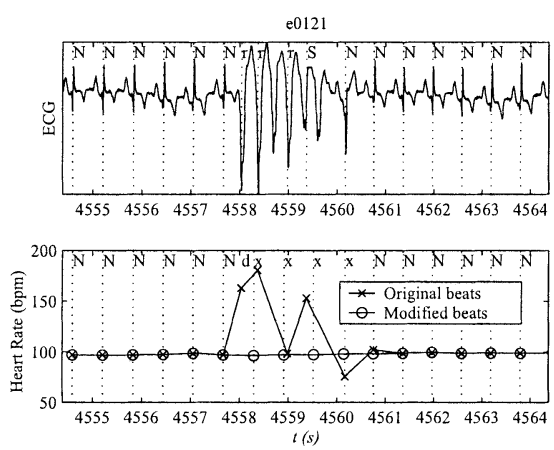

(f)

Fig. 1. Different kinds of anomalies in the ESDB database. Upper panel represents the ECG with the originally annotated beat classification. Lower panel shows the original heart rate and the modified one indicating which action has been carried out. (a) FP deletion. (b) FN insertion. (c) Supraventricular ectopic beat moving. (d) Ventricular ectopic beat moving. (e) Deletion and insertion in one-step double correction. (f) Multiple insertion of evenly spaced beats.

HP or heart rate HR or low-pass filtered event series (LPFES). The continuous time generalization of the IPFM model can be written as

$$
x=\int_{0}^{t(x)} \frac{1+m(\tau)}{T} d \tau=\int_{0}^{t(x)} r(\tau) d \tau
$$

where $t(x)$ is a continuous function that solves the model equation and whose values at $x=k=1,2, \ldots, N\left(t(k)=t_{k}\right)$ are the $k$ th beat occurrence time. $(1+m(t)) / T$ can be seen as the instantaneous heart rate $r(t)$. $T$ is the mean $\mathrm{RR}$ interval in the analyzed period and $m(t) / T$ represents the dynamic part of the instantaneous heart rate, which is zero-mean. The dynamic part is usually small compared with the mean heart rate $(m(t) \ll 1)$. The first beat is considered to occur at $t_{0}=0$ and $m(t)$ is assumed to be causal, hence, $m(t)=0$ if $t<0$. Another important restriction imposed on the $m(t)$ signal is that it is a band-limited signal with negligible power spectral density over $0.4 \mathrm{~Hz}$.

The heart timing $(h t)$ signal is also introduced in [8] as the integral of the modulating signal

$$
h t(t)=\int_{0}^{t} m(\tau) d \tau
$$

and, from (2), we obtain

$$
h t(t(x))=x T-t(x) .
$$

This $h t$ signal defined in (3), and observable at $t_{k}$ according to (4), is then used to estimate the PSD of the HRV, since $M(\omega)=$ $\mathcal{F} \mathcal{T}(m(t))$ can be estimated as $\hat{M}(\omega)=j \omega \cdot H T(\omega)$.

An ectopic electrical impulse may cause the premature resetting of the integration process on the SA node, giving a phaseshifted series of normal beats following the ectopic beat. If the ectopic beat is the $k_{e}$ th, it can be interpreted that the beats preceding the ectopic beat are given at $t_{k}=t(k)$ with $k$ integer and $k<k_{e}$, and the beats subsequent to the ectopic beat are given at $t_{k}=t(k-1+s)$ with $k$ integer and $k>k_{e}$. The premature resetting of the integration occurs at $t\left(k_{e}-1+s\right)$ and $s$ is an unknown real quantity $(s \leq 1)$ corresponding to the value reached for the integral at the resetting time $t\left(k_{e}-1+s\right)$. It is important not to confuse the unknown $t\left(k_{e}-1+s\right)$ instant with the known (estimate) ectopic beat occurrence time, because the propagation mechanisms of the ectopic impulse may be somewhat different to the normal beat and then, the instant of the SA node reset $\left(t\left(k_{e}-1+s\right)\right)$ will not be the same as the one associated with the ectopic beat position, typically from a QRS detector. The time occurrence of the ectopic beat has no relation to the SA node and it will not be used by the proposed method.

Fig. 2 shows the signals involved in this IPFM extension. The dashed curve represents the $x$ function given by (2). The continuous curve is the same function but reset when it reaches the corresponding threshold. The normal threshold is one but the ectopic beat prematurely resets the integral process at $s$. The subsequent beats will correspond to values of $x$ coming from integers to which have been added an unknown constant magnitude $s$. 


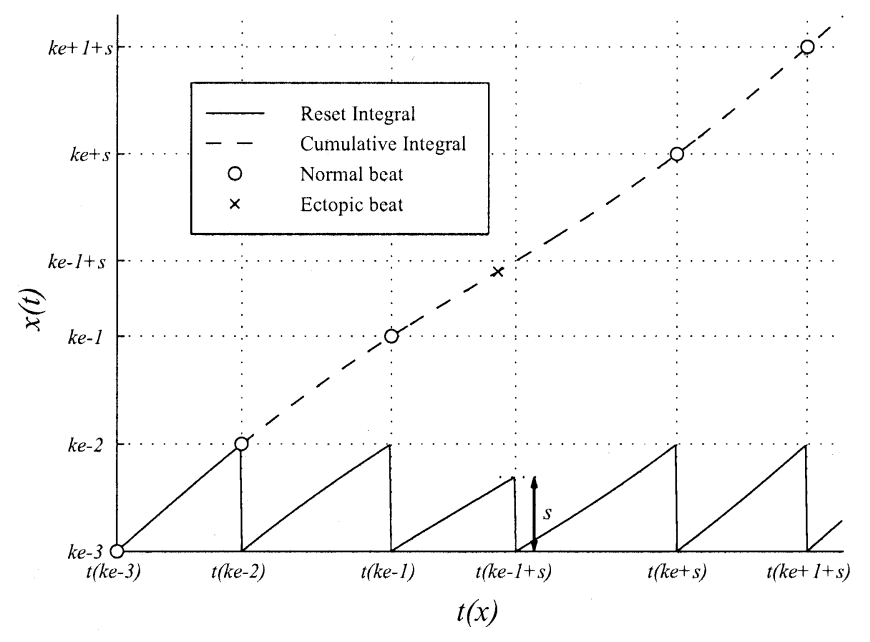

Fig. 2. Beat occurrence time generation with ectopic beat presence from the integral of the IPFM model. See text for details.

From this interpretation of ectopic beats, (4) can be estimated before and after the ectopic beat if the $s$ and $T$ values were known. The correct values of $h t(t)$ at each beat occurrence time can then be calculated. Explicitly, in a series of $N$ beats with an ectopic beat detected at the $k_{e}$ position the correct values of the HT signal for the beats preceding and following the ectopic beat will be

$$
\left\{\begin{aligned}
h t\left(t_{k}\right) & =h t(t(k))=k T-t(k) & & \\
& =k T-t_{k}, & & \text { for } k>k_{e} \\
h t\left(t_{k}\right) & =h t(t(k-1+s)) & & \\
& =(k-1+s) T-t(k-1+s), & & \text { for } k>k_{e} \\
& =(k-1+s) T-t_{k} & &
\end{aligned}\right.
$$

where the instants $t_{k}$ with $k<k_{e}$, and $k>k_{e}$, are the known beat occurrence times before and after the ectopic beat, respectively. If there are several ectopic beats, the jump $s$ for each ectopic beat and the correct $T$ needs to be known to calculate the HT values. The section below describes the method for determining these a priori unknown $s$ and $T$ quantities required to estimate $h t\left(t_{k}\right)$ according to (5).

To understand the role of the $s$ quantity it should be noted that it represents a phase shift in the integration process of the IPFM model (premature resetting). It is not difficult to see that the beat timing after the ectopic beat is not a delayed version of the beat timing that would have occurred if the ectopic beat had not occurred. Dealing with ectopic beats correction by introducing a constant delay in the beat timing cannot then correctly recover the beat sequence. At best, the delay could only absorb the spike at the ectopic beat position but this will result in a phase shift in the HT or other HRV signals before and after of the ectopic beat, and consequently, the spectra will continue to be corrupted. Something similar will happen when substituting the ectopic beat by inclusion of an intermediate beat. This will create two false, shortened RR intervals [see Fig. (1c)].

This simple IPFM-based model for anomalies in beat occurrence modeling does not intend to explain the complex physiology of ectopic beat generation but it can explain disturbances between the normal beats adjacent to the anomaly whatever the cause might have been. A safe interpretation of the jump $s$ is as follows: $s=1$ means that something occurred (probably a false negative or a ventricular ectopic beat) that did not reset the process of beats generation [see Fig. 1(b), (d)], $s<1$ means that something occurred (probably a supraventricular ectopic beat) that reset the process of beats generation prematurely [see Fig. 1(c)] and $s>1$ means that several consecutive normal beats were missed due to ectopic beats or false negatives [see Fig. 1(e), (f)]. False positives are simply corrected by deleting them [see Fig. 1(a)]

2) Instantaneous Heart Rate and HT Determination: The starting point of this problem is the known occurrence times of the normal beats before, $t_{k}=t(k)$ with $k<k_{e}$, and after the ectopic beat, $t_{k}=t(k-1+s)$ with $k>k_{e}$. Using these values, the samples of the HP signal, $h p\left(t_{k}\right)=t_{k}-t_{k-1}$, can be calculated, taking into account that all values obtained will be correct except for those involving the ectopic beat. So, it can be seen, for the beats before the ectopic beat, the values of the HP signal are $h p(t(k))=t(k)-t(k-1)$ with $k<k_{e}$, and for the beats after the ectopic beat $h p(t(k-1+s))=t(k-1+s)-t(k+s-2)$ with $k>k_{e}+1$. A sharp impulse at $t\left(k_{e}+s\right)=t_{k_{e}+1}$ is obtained, having a value $t\left(k_{e}+s\right)-t\left(k_{e}-1\right)$. This incorrect value must be rejected before any further assessment. Using only the correct values of $h p(t)$ allows a continuous time estimation $\widehat{h p}(t)$ by means of spline interpolation. ${ }^{1}$

Once estimated $\widehat{h p}(t)$, the virtual beat times $t\left(k_{e}-n+s\right)$, being $t\left(k_{e}-n+s\right)$ the hypothetical $n$th normal beat positions backward extended prior to the first normal beat after the ectopic beat $\left(t\left(k_{e}+s\right)\right)$, can be calculated as

$$
t\left(k_{e}-n+s\right)=t\left(k_{e}-n+1+s\right)-\widehat{h p}\left(t\left(k_{e}-n+1+s\right)\right) .
$$

This equation is evaluated from $n=1$ up to $t\left(k_{e}-n+s\right)<$ $t\left(k_{e}-1\right)$. At this point, the backward extended beat sequence is overlapped in time with the existing beat sequence previous to the ectopic beat (see Fig. 3).

For the existing beat sequence previous to the ectopic beat, the continuous function $x(t)$ is estimated by interpolating the known pairs $(k, t(k))$ for $k=1,2, \ldots k_{e}-1$ and can also generate a forward extended function, $\widehat{x}^{f}(t)$. Similarly, for the backward extended beat sequence, the continuous function $x_{s}(t)=x(t)-s$ is estimated by interpolating the known pairs $(k, t(k+s))$ for $k=k_{e}-n, \ldots, N$, giving $\widehat{x}_{s}^{b}(t)=\widehat{x}^{b}(t)-s$. Once both curves have been estimated, the vertical distance between them is the desired value of $s$. The curve displacement can be calculated at any point, or even the area between both curves divided by the intersection time allows for the estimation of $s$ as

$$
\widehat{s}=\frac{1}{t\left(k_{e}\right)-t\left(k_{e}-1+s\right)} \int_{t\left(k_{e}-1+s\right)}^{t\left(k_{e}\right)}\left(\widehat{x}^{f}(t)-\widehat{x}_{s}^{b}(t)\right) d t .
$$

Fig. 3 shows both functions for the same case of Fig. 2 and also shows the original normal beats and the calculated extended

\footnotetext{
${ }^{1}$ High-order splines are used to minimize the low-pass filtering effect of the interpolation [8]. Also, it was shown that directly using the $\widehat{h p}(t)$ signal or the interpolated HR signal for PSD estimate of HRV there appears a spectral corruption due to the nonlinear relationship between these signals and the instantaneous heart rate $r(t)$.
} 


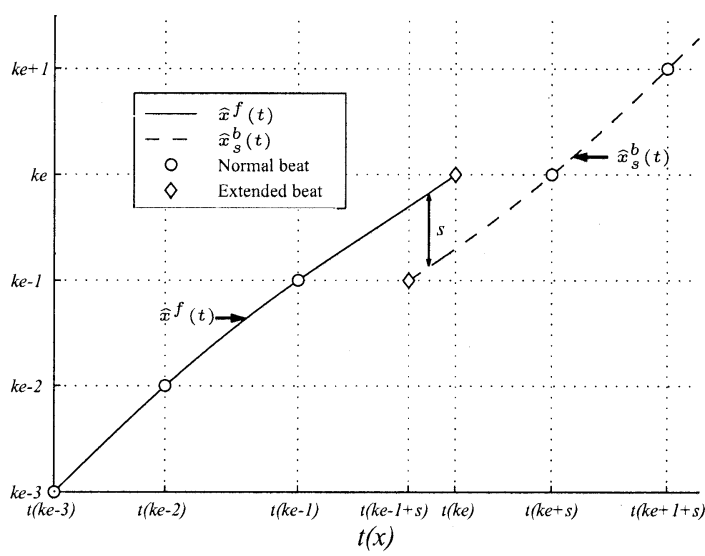

Fig. 3. Forward and backward extended beat occurrence times and the interpolated continuous curves can be calculated from the known normal beats. Note that the offset of the backward extended curve is $s$ after the ectopic beat. Circles represent the previously known beat occurrence times and diamonds represent the forward and backward calculated beat occurrence times.

beats. This process must be repeated once for each ectopic beat to obtain their corresponding $\widehat{s}$ value.

The mean heart period $T$ must still be estimated to evaluate $h t\left(t_{k}\right)$ from (5). If there have not been ectopic beats, $T$ would be $T=t(N) / N$, where $N$ is the final beat order $(\mathrm{N}+1$ total beats). In the presence of ectopic beats, the same calculation can be made, taking into account that the effective number of beats will be $N+1+\sum_{j} \widehat{s}_{j}$, where $\sum_{j} \widehat{s}_{j}$ is the sum of all jumps at each $j$ th ectopic beat and that the last beat occurrence time is $t_{N}=t\left(N+\sum_{j} \widehat{s}_{j}\right) \neq t(N)$. Thus, $T$ will be

$$
T=\frac{t_{N}}{N+\sum_{j} \widehat{s}_{j}}
$$

With the estimated $\widehat{s}_{j}$ and $T$ values, (5) can be used to estimate the HT signal at the irregularly spaced beat times $t_{k}$ and then, by spline interpolation, to obtain a continuous estimate of the HT signal. Finally, the $m(t)$ signal can be obtained in the time domain as

$$
m(t)=h t^{\prime}(t)
$$

or its spectrum in frequency domain as

$$
M(\omega)=j \omega \cdot H T(\omega)
$$

\section{Controlled Evaluation Protocol}

To evaluate and compare the behavior of the spectral estimates in the presence of ectopic beats, we generate a controlled $m(t)$ signal. This $m(t)$ is the output of an AR model driven by white noise. The transfer function of the AR model approximately matches a typical PSD at supine rest, being bandlimited to $0.4 \mathrm{~Hz}$ as described in [8]. Fifty random estimates of $m(t)$ were generated at a sampling rate of $1 \mathrm{~Hz}$ (1024 s length), giving $m[n]$, according to the PSD of the AR model. Then, the $m[n]$ sequences are interpolated by a factor 128 in order to more precisely calculate the cumulative integral in the IPFM model. To generate the beat occurrences, the IPFM model (see Fig. 2) is driven by $(1+m[n]) / T$ and the cumulative integral is calculated, obtaining the sequence of the beat times, $t_{k}$, as the instants when the integral crosses $k+\sum_{j=1}^{l(k)} s_{j} \cdot u\left(k-k_{e_{j}}\right)$, as

$$
k+\sum_{j=1}^{l(k)} s_{j} \cdot u\left(k-k_{e_{j}}\right)=\int_{0}^{t_{k}} \frac{1+m(t)}{T} d t
$$

where $u(k)$ is the unitary step function, $k_{e_{j}}$ are the ectopic beat order artificially introduced by early resetting of the integral, and $l(k)$ is the number of ectopic beat prior to the $k$ th beat.

In this fashion, a total of $M$ jumps of magnitude $s_{j}$, with $j=1, \ldots, M$ are generated to simulate the beat occurrence times with the presence of $M$ ectopic beats located at random positions $k_{e_{j}}$. For each generated realization of $m[n]$, a variable number of jumps $M$ is introduced, using $M=[0,1,2,3,4,6,8,12,16,20]$ as well as three different $s$ values, $s=[0.6,0.8,1]$. This selected range of $s$ values is common enough in real situations since $s>1$ usually means lost beats and $s<0.6$ is infrequent due to the refractory period of the heart cells. In all cases, $T=1 \mathrm{~s}$ (mean heart rate of 60 $\mathrm{bpm}$ ), is used. Of course, these values are known during the beat generation phase but they are supposedly unknown, and must be estimated during the PSD analysis process. Finally, the PSD estimation to recover the PSD of the modulating signal $m(t)$ is made by the following methods of HRV PSD estimation.

- DFT of the interpolated HT signal (FHTIS): Following the described method of determining the jumps $\left(s_{j}\right)$, it is estimated $h t\left(t_{k}\right)$. Posterior fourteenth-order spline interpolation generates a continuous $h t(t)$, later evenly sampled at regular time intervals. Then, the DFT is applied to obtain the PSD estimation according to (10) and squaring.

- DFT of the interpolated HP and HR signals (FHPIS, FHRIS): Considering that the signals based on differences, such as the HP and HR, only present incorrect values at the ectopic beat times, these signals are evaluated by spline interpolation of fourteenth order with the incorrect values previously removed. Then, the DFT is applied to obtain the PSD estimate as explained before.

- Lomb method of the HP and HR signals (LHP, LHR): The Lomb-Scargle periodogram is used [20], [21] as representative of the direct spectral estimation methods and one that estimates the PSD of a unevenly sampled signal without need of interpolation. The method is applied as in the preceding case to the HP and HR signals with the values at each ectopic beat previously removed.

- Spectrum of Counts with filling (SPC-F): The spectrum of counts [15] obtains the information directly from the beat occurrence times. Provided that there is no intermediate time-domain signal, the ectopic-created gaps between beats can be corrected only by filling the gap or by shifting all the successive beats to cover up the gap. If the gaps due to the ectopic beats are not covered, the spectrum obtained will be corrupted, even in the case of a single ectopic beat. With this method, the gaps are filled by a hypothetical beat in the middle of each gap, and then the spectrum of counts method is applied to this sequence. 
- Spectrum of Counts with shifting (SPC-S): In this case, the subsequent beats after each ectopic beat are shifted to maintain RR intervals between beats that always belongs to inter SA beats distance. Then, the spectrum of counts method is applied.

The PSD of each $i$ th realization of the $m[n]$ sequence ( $\left.m_{i}[n]\right), P S D_{i}(f)$ measured as the squared DFT of $\left(m_{i}[n]\right)$, is taken as the objective to be estimated. Each trial is considered as an independent recording for estimating its PSD and this spectrum is termed the "original spectrum" of each realization. The global behavior of each method was evaluated by calculating the normalized error power (NEP) defined as

$$
\mathrm{NEP}_{i}=\frac{\int_{-\frac{1}{2 T}}^{+\frac{1}{2 T}}\left|\widehat{\operatorname{PSD}}_{i}(f)-\operatorname{PSD}_{i}(f)\right| d f}{\int_{-\frac{1}{2 T}}^{+\frac{1}{2 T}} \operatorname{PSD}_{i}(f) d f}
$$

where $\widehat{\mathrm{PSD}}_{i}(f)$ is the HRV PSD estimate with each one of the methods on the $i$ th realization $m_{i}[n]$. The mean of the $\mathrm{NEP}_{i}$, (MNEP) is calculated for the total 50 realizations.

The calculated MNEP is an effective index of the quality of the PSD estimation methods. However, in HRV the PSD is usually divided into different frequency bands with different clinical information. This bands are typically those recommended in [22]: VLF $(0.003-0.04 \mathrm{~Hz}), \mathrm{LF}(0.04-0.15 \mathrm{~Hz})$, and $\mathrm{HF}(0.15-0.4 \mathrm{~Hz})$. The most common clinical indexes are based on the power in each band relative to the sum of the power in the three bands. The relative power VLF/AF, LF/AF, and $\mathrm{HF} / \mathrm{AF}$ are usually calculated, where $\mathrm{AF}=\mathrm{VLF}+\mathrm{LF}+\mathrm{HF}$ and VLF, LF, and HF are the power in the corresponding band. The errors in each band are then also calculated as performance parameters. The difference of the relative power obtained with each method and that obtained from the original realization $m_{i}[n]$ is defined as

$$
\begin{aligned}
E_{\mathrm{VLF}} & =\frac{\widehat{\mathrm{VLF}}}{\widehat{\mathrm{AF}}}-\frac{\mathrm{VLF}}{\mathrm{AF}} \\
E_{\mathrm{LF}} & =\frac{\widehat{\mathrm{LF}}}{\widehat{\mathrm{AF}}}-\frac{\mathrm{LF}}{\mathrm{AF}} \\
E_{\mathrm{HF}} & =\frac{\widehat{\mathrm{HF}}}{\widehat{\mathrm{AF}}}-\frac{\mathrm{HF}}{A F}
\end{aligned}
$$

These errors are signed magnitudes whose sum in the three bands is zero. If the error is positive in one band, the relative estimated power is excessive in this band and too small if negative. The tendency of each method to increase or decrease one band power with respect to others can readily be detected. The mean and standard deviation of this error are also evaluated.

\section{Actual Data Evaluation Protocol}

In real situations, the PSD of the HRV is unknown and it is difficult to demonstrate which method is better than others. However, an experiment has been developed to evaluate the proposed method also in real cases. We have defined three time segments in records containing ectopic beats: The first segment (A) is a 4-min period just before of an ectopic beat sequence and it must

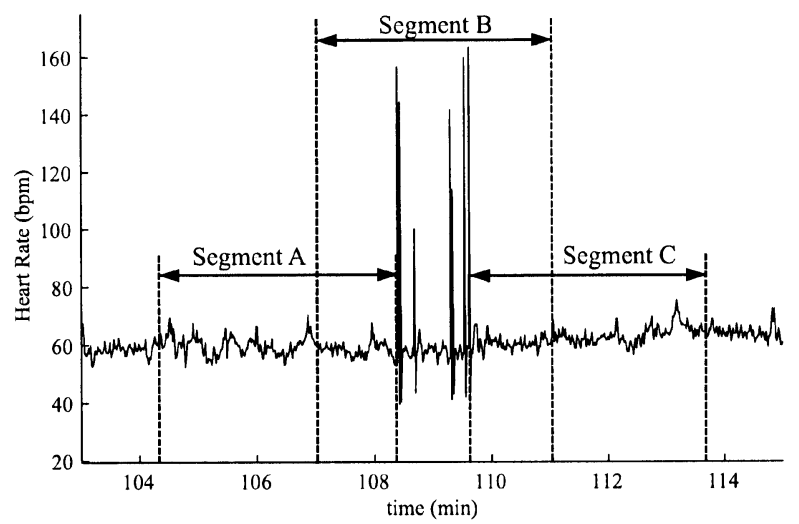

Fig. 4. Definition of the time segments $\mathrm{A}, \mathrm{B}$, and $\mathrm{C}$ for the actual data evaluation protocol.

be free of ectopic beats. The second segment (B) is a 4-min period centred at the ectopic beat sequence that includes one or several ectopic beats. The third segment $(\mathrm{C})$ is a 4-min period just after the ectopic beat episode and it must be ectopic beats free. Fig. 4 shows these period definitions on an HR series. This segmentation has been applied to the ESDB database giving 132 valid ectopic beat episodes with four minutes ectopic beat free before and after the ectopic beat episode. In the 132 cases there are 91 with one isolated ectopic beat, 28 with two, five with three, four with four, two with eight, and two with ten ectopic beats.

It is assumed that the HRV should be stationary during the time including the three A, B, C periods. So, it will be possible to compare the ectopic-beat-free behavior in A, C with the ectopic beat treated behavior in B.

The FHRIS and FHTIS methods, defined in the previous section, have been applied to determine the spectral power at the LF and HF bands in each segment. Then, a paired samples statistical study is performed to determine significative differences between methods and between segments A, B, and C. So, to determine differences between methods we define six variables $\triangle H T H R_{X F}^{S}$ as the difference between the power at the LF band $(X=L)$ and HF band $(X=H)$ with the FHTIS method and with the FHRIS method during segment $S(S=A, B, C)$. To determine differences between segments with both methods we define 12 variables, $\Delta H Q_{X F}^{S S^{\prime}}$, as the difference between the spectral power during the segment $S$ minus that obtained during segment $S^{\prime}$ ( $S$ and $S^{\prime}$ substituted by $A, B$, or $C$ ) at the LF band $(X=L)$ and $\mathrm{HF}$ band $(X=H)$ with the method $Q(Q=R$ for FHRIS and $Q=T$ for FHTIS). The mean, standard deviation, and significance test of each variable has been carried out.

\section{RESULTS}

\section{A. Threshold for the Detection of Anomalous Beats}

To obtain a suitable threshold $(U)$ in (1), a wide range of different recordings was analyzed. So, the left-hand side of (1) was measured in percutaneous transluminar coronary angiography (PTCA) recordings from the STAFF III database [23], in recordings from the European ST-T database (ESDB) [12], in recordings from healthy subjects and during episodes of atrial fibrilation from the MIT-BIH database. To illustrate that, $\left|\widehat{r_{k}^{\prime}}\right|$ 

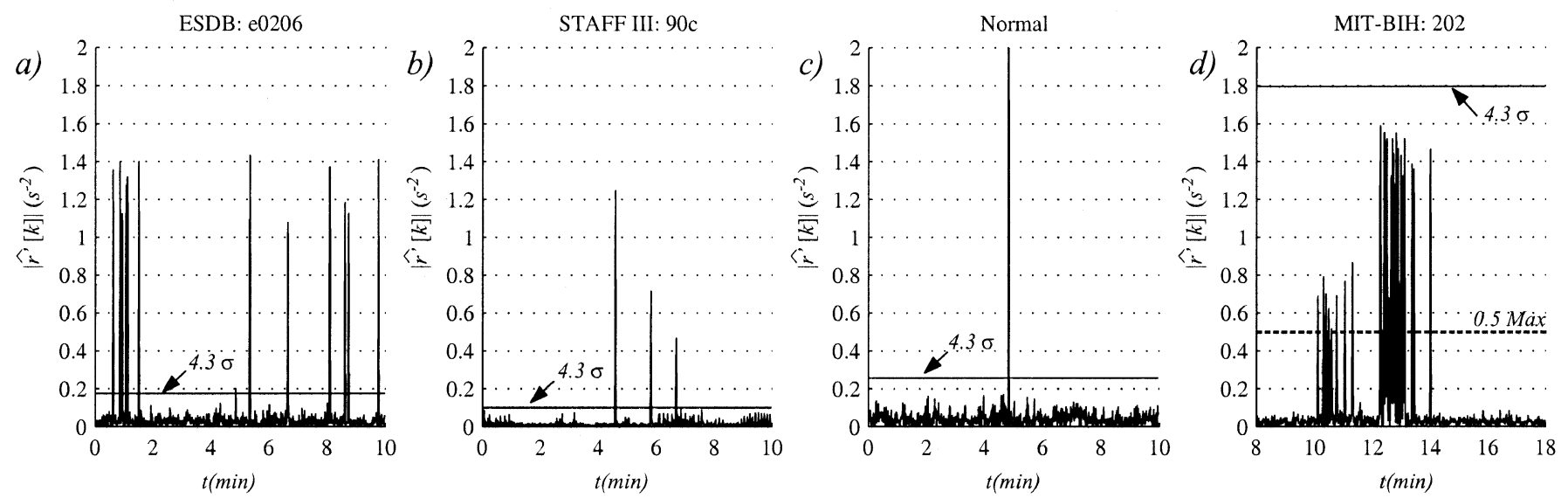

Fig. 5. Derivative of the instantaneous heart rate estimation from the records "e0206" of the ESDB, 90c from the Staff III database (PTCA), from a normal subject and from the record 202 of the MIT-BIH database with atrial fibrilation. The selected threshold $U=4.3 \sigma_{\widehat{r_{k}^{\prime}}} s^{-2}$ is shown on each panel.

has been represented in Fig. 5 for some of these records. The selected records are representatives of the general behavior of each group.

The derivative of the heart rate estimated in (1) is sensitive enough to distinguish an FP or a FN from normal values (see Fig. 5). Also, in most cases of ectopic beats, the value of $\left|\widehat{r_{k}^{\prime}}\right|$ is large enough to detect the anomaly. However, a QRS detector misplacement can be more difficult to detect as it occurs around the minute five on Fig. 5(a). So, a tailored threshold $U$ may be calculated as function of the standard deviation of $\left|\widehat{r_{k}^{\prime}}\right|\left(\sigma_{\widehat{r_{k}^{\prime}}}\right)$ as $U=\xi \cdot \sigma_{\widehat{r_{k}^{\prime}}}$. The value of $\xi$ can be optimized in terms of the sensitivity and the specificity achieved by the method. Fig. 6 represents the receiver operating characteristic (ROC) curve of the method applied to the mentioned databases for values of $\xi$ varying from 3.0 to 6.0 in steps of 0.1 . The obtained $\xi$ value that better balances the sensitivity and the specificity is $\xi=4.3$ ( sensitivity $=96.4 \%$, specificity $=96.8 \%$ ).

Nonstationary recordings may require a time-variant threshold. In these cases, the standard deviation may be evaluated during a long term (more than $5 \mathrm{~min}$ ) moving window. In any case, an upper limit of $U=0.5 \mathrm{~s}^{-2}$ must be imposed to reject possible episodes of atrial fibrillation or other arrhythmias as shown in Fig. 5(d). From our experience, this limit is largely exceeded during typical arrhythmias but is large enough to not be reached by normal beats.

\section{B. Simulation Results}

Fig. 7 presents a summary of the main simulation results. The first column shows the MNEP, respectively, of the seven different methods as a function of the number of ectopic beats and for each $s$ value (50 realizations for each $s$ ). The second column presents the mean of the error in each band, joining the realizations with different $s$ values (150 realizations) and the third column shows the standard deviation of the same errors. A signed stacked bar was used to represent the different magnitudes; the total amplitude of the bar representing the total mean error and each shaded area represents the contribution of each band; the white area is for the VLF band, the gray one is for the LF band and the darkest one is for the HF band.

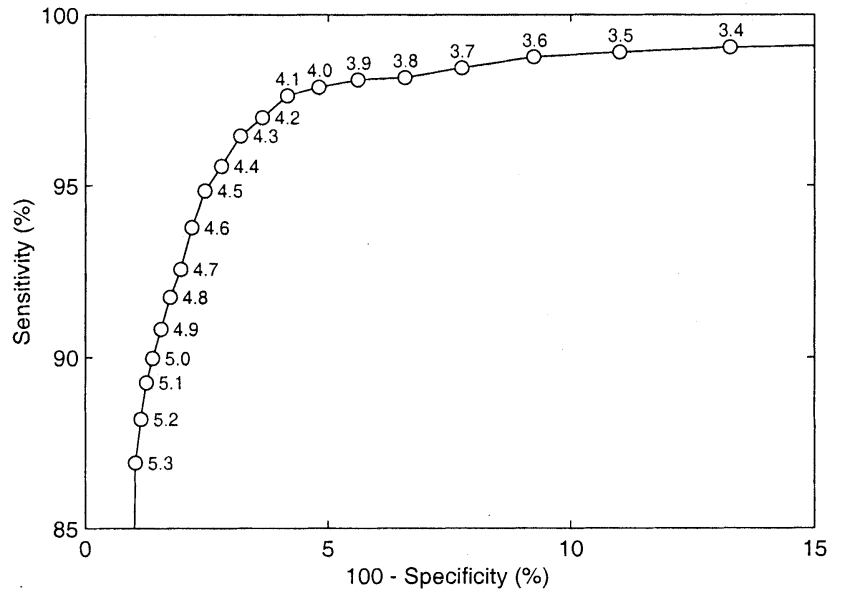

Fig. 6. ROC curve for values of $\xi$ varying from 3.0 to 6.0 in steps of 0.1 .

From the analysis of the MNEP curves, it can be observed that the FHPIS, FHRIS, LHP, and LHR methods maintain a biased error due to the election of the HP or HR signals as representatives for the HRV. This error (when $M=0$ ) is inherent to the time-domain signal and does not depend on the number of ectopic beats. The differences between HR or HP are small, being favorable to FHRIS with respect to FHPIS, and LHP with respect to LHR, but the performance is similar. This result was previously pointed out in [8]. For the dependence with the number of ectopic beats, the four methods mentioned exhibit a quasi-linear dependence, with larger slopes when the jump magnitudes ( $s$ values) are larger. The SPC-based methods show how their appropriate performance without ectopic beats is quickly lost in the presence of ectopic beats. The SPC with filling is the only method that performs better with larger $s$ values ( $s$ values close to one). This was expected, as filling with a new beat offers a better solution when $s=1$. In spite of this, none of the SPC-based results present a good fit to the original spectrum. Finally, the FHTIS has the best performance without ectopic beats, $\left(\operatorname{MNEP}_{\mathrm{FHTIS}}(0)=0.37 \cdot 10^{-3}\right.$ with respect to the closest method in this comparison, SPC, $\left(\operatorname{MNEP}_{\mathrm{SPC}}(0)=1.45 \cdot 10^{-3}\right.$, and also the least sensitivity to 

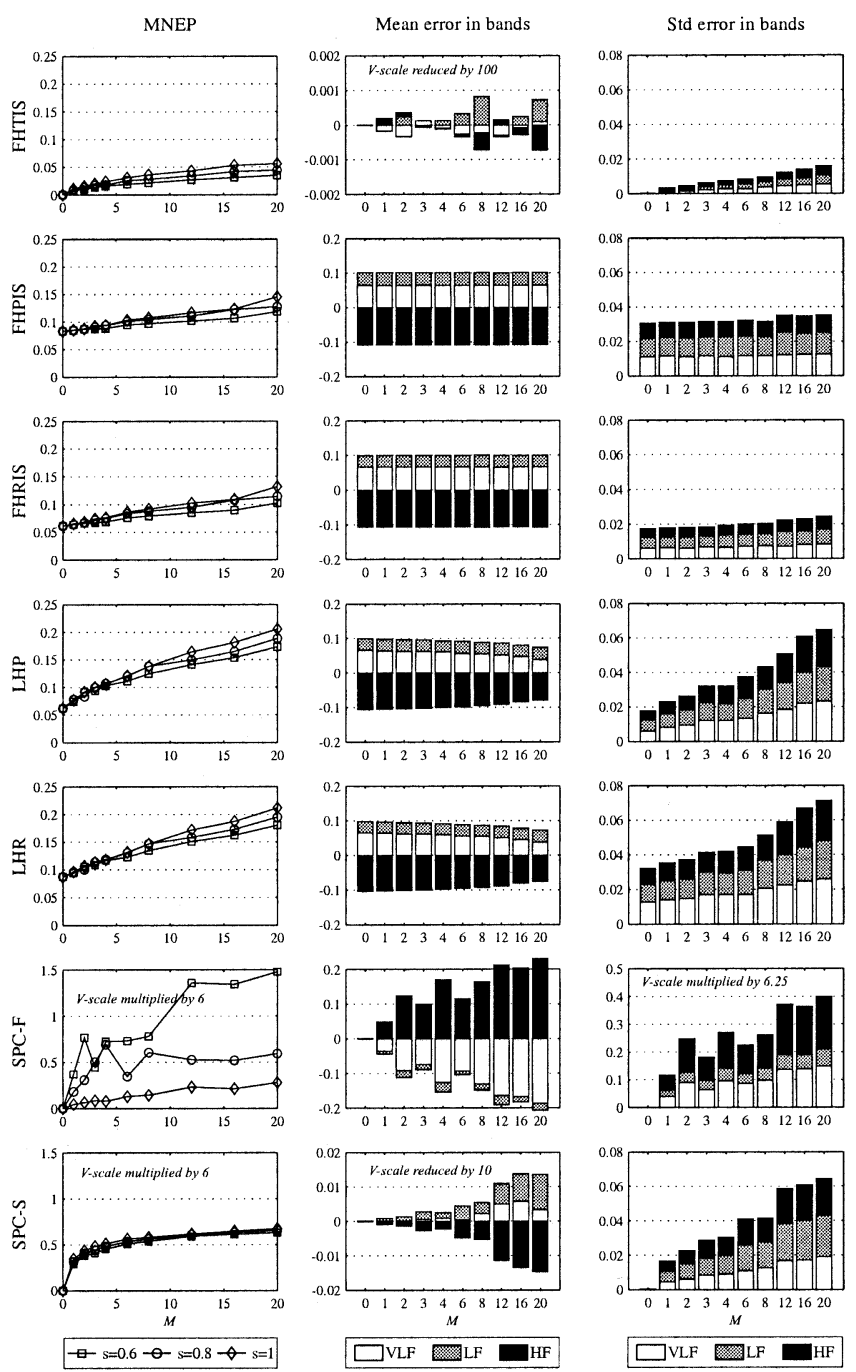

Fig. 7. MNEP of each method as a function of $M$ and $s$ (50 realizations for each $s$ value). Mean and standard deviation of the relative error in the VLF, LF, and $\mathrm{HF}$ bands (150 realizations joining together the different $s$ values). Note the different vertical scales (V-scales) in several plots.

the ectopic beats measured as the slope $(\alpha)$ of the MNEP curve $\left(\alpha_{\text {FHTIS }}=2.6 \cdot 10^{-3}\right)$ with respect to the closest method performance in this comparison, FHPIS, $\left(\alpha_{\text {FHPIS }}=2.8 \cdot 10^{-3}\right)$. However, since the HT ectopic beat treatment uses the interpolation of the HP signal as departure point, it can be stated that the ectopic beat influence is similar in both cases with HT being more desirable because of the exact reproduction of the $m(t)$ signal in situations without ectopic beats. The HT is, thus, a better alternative since it does not exhibit an inherent residual error as the HP or HR signals do.

Analysis of the mean and standard deviation of the error in each band shows that the SPC method with shifted beats (SPC-S) performs much better when the clinical indexes are analyzed by integrating the PSD in large bands (see the middle column of Fig. 7) than when the spectrum is examined directly (see the left column of Fig. 7). So, this method does not keep an accurate detailed shape of the spectrum but keeps the power at each band well maintained. However, the error is still one order of magnitude higher than in the method based on the HT signal. Most of the methods exhibit a low-pass filtering effect except for the SPC fill method that has a strongly increased power at the HF band. The FHTIS has no noticeable filtering effect. The curious decrease of the mean error with the number of ectopic beats in the LHP and LHR methods is due to the presence of the ectopic beat that increases the high-frequency power and compensates the low-pass filtering effect introduced by the HP or HR signals themselves. Even more important, in real situations result the fact that in all methods the standard deviation is larger than that obtained by the FHTIS method. This fact indicates an additional variance due to the method that in some situations may mask a possible diagnosis. The standard deviation of the error with the FHTIS method is lower by about one order of magnitude with respect to the method with lower bias (SPC-S) and also is lower than that obtained by the next best method in terms of standard deviation (FHRIS), particularly when the number of ectopic beats is small.

\section{Real Data Results}

Table I shows the differences between the clinical indexes at the LF and HF bands by means of the FHTIS and FHRIS methods during the segments $\mathrm{A}, \mathrm{B}$, and $\mathrm{C}$ defined in Section II-D. During the segments without ectopic beats (A and $\mathrm{C}$ ), the FHTIS method obtains more power at LF and particularly at the HF band. These differences were statistically significant. This is the expected behavior since the FHRIS method has a low pass filtering effect [8]. However, during the segment with ectopic beats (B), much more power at both bands was estimated by means of the FHRIS method but with a large standard deviation, large enough to make the differences statistically not significant. This behavior is due to the great dispersion of the estimation of the clinical indexes estimates achieved by the FHRIS method (standard deviation during B with FHRIS was 14.7 times larger than with FHTIS at the LF band and 7.2 times larger at the HF band, considering all cases together).

Table II shows the differences between segments with both methods. It can be observed that when the B segment is involved, the mean and standard deviation is much larger than with the FHRIS method. Again, this fact indicates a large dispersion in the estimates with the FHRIS method. However, the FHTIS method obtains smaller mean differences but much smaller standard deviations, so much that this method finds significant differences between segments. So, the B segment presents more power than A or C, particularly at the LF band. This finding corroborates that pointed out in [3] and it must be further investigated with a more elaborated procedure since this was not an objective of the present paper but it serves to show how dispersion may mask a possible physiological behavior.

Under the assumption of the stationary HRV across segments $\mathrm{A}, \mathrm{B}$, and $\mathrm{C}$, no differences should be found when comparing $\mathrm{A}$ and $\mathrm{C}$. The differences found have a mean error of $30 \mathrm{~ms}^{-2}$ for LF band and about $20 \mathrm{~ms}^{-2}$ for HF band (see Table II), giving the limits of stationarity for these data. If the differences between $\mathrm{A}$ and $\mathrm{B}$, and also between $\mathrm{B}$ and $\mathrm{C}$, are in this range it means that the ectopic beat correction method gives acceptable results. The FHTIS method gives about $100 \mathrm{~ms}^{-2}$ for LF 
TABLE I

Mean and Standard DeViation of the Differences BetweEn the Clinical IndeXes at the LF and HF Bands by MEANS OF tHe FHTIS and FHRIS Methods During the Segments A, B, AND C. Significant DifFerenCes ARE BoldFaced $(p<0.05)$

\begin{tabular}{c|c|c|c|c|c|c}
\hline \hline Band & \multicolumn{3}{|c|}{ LF } & \multicolumn{3}{c}{ HF } \\
\hline Variable & $\Delta H T H R_{L F}^{A}$ & $\Delta H T H R_{L F}^{B}$ & $\Delta H T H R_{L F}^{C}$ & $\Delta H T H R_{H F}^{A}$ & $\Delta H T H R_{H F}^{B}$ & $\Delta H T H R_{H F}^{C}$ \\
\hline Mean $\left(\mathrm{ms}^{-2}\right)$ & $\mathbf{1 3}$ & -1172 & $\mathbf{1 2}$ & $\mathbf{6 8}$ & -205 & $\mathbf{7 1}$ \\
Std $\left(\mathrm{ms}^{-2}\right)$ & $\mathbf{1 7}$ & 11491 & $\mathbf{1 8}$ & $\mathbf{6 2}$ & 2187 & $\mathbf{6 9}$ \\
\hline \hline
\end{tabular}

TABLE II

Mean and Standard Deviation of the Differences Between Segments With FHTiS and FHRis Methods. SIGNIFICANT DiFFERENCES ARE BOLDFACED $(p<0.05)$

\begin{tabular}{c|c|c|c|c|c|c|c|c|c|c|c|c}
\hline \hline Band & \multicolumn{9}{|c|}{$\mathrm{LF}$} & \multicolumn{8}{c}{$\mathrm{HF}$} \\
\hline Variable & $\Delta H T_{L F}^{A B}$ & $\Delta H R_{L F}^{A B}$ & $\Delta H T_{L F}^{B C}$ & $\Delta H R_{L F}^{B C}$ & $\Delta H T_{L F}^{A C}$ & $\Delta H R_{L F}^{A C}$ & $\Delta H T_{H F}^{A B}$ & $\Delta H R_{H F}^{A B}$ & $\Delta H T_{H F}^{B C}$ & $\Delta H R_{H F}^{B C}$ & $\Delta H T_{H F}^{A C}$ & $\Delta H R_{H F}^{A C}$ \\
\hline Mean $\left(\mathrm{ms}^{-2}\right)$ & -71 & -1256 & $\mathbf{1 0 3}$ & 1288 & 32 & 31 & $\mathbf{- 5 8}$ & -331 & $\mathbf{3 8}$ & 314 & -20 & -17 \\
Std $\left(\mathrm{ms}^{-2}\right)$ & 575 & 11767 & $\mathbf{4 1 5}$ & 11657 & 670 & 658 & $\mathbf{2 1 3}$ & 2323 & $\mathbf{1 7 7}$ & 2292 & 186 & 140 \\
\hline \hline
\end{tabular}

band compared with $1000 \mathrm{~ms}^{-2}$ that the FHRIS method obtains. In the HF band FHTIS gives about $40 \mathrm{~ms}^{-2}$ compared with $300 \mathrm{~ms}^{-2}$ obtained by the FHRIS method. These numbers corroborate one order of magnitude differences in preserving the original clinical indexes in favor of FHTIS method compared with the next best FHRIS method from the simulation section.

\section{CONCLUSION}

This paper presents a study analysing the problems of ectopic beats on the estimation of the PSD in HRV. First, an algorithm has been presented to identify the anomalies in the heart rate (ectopic beats or false QRS detections). The continuous time IPFM model has been extended to model the resetting of the SA node when ectopic beats appear and it is found that the heart timing signal continues to be an adequate representation of the HRV when proper ectopic beat processing is done. A method is proposed for correctly determining the heart timing signal, thus, overcoming the problem caused by the presence of ectopic beats. The results compare most of the commonly used PSD estimation methods in the presence of ectopic beats. These results show how the method presented in this paper, based on the heart timing signal, brings improved behavior. The obtained mean error in clinical bands is ten times better and the obtained standard deviation is five times better than the next best method in simulation and more than ten times in actual data. The satisfactory performance of the SPC method without ectopic beats is quickly lost with the presence of ectopic beats. The other methods start with a poorer performance, even when there are no ectopic beats.

In summary, the presence of ectopic beats strongly reinforces the value of using the heart timing signal together with the proposed ectopic beat treatment. The IPFM model can be adapted to model the presence of ectopic beats and from this perspective the time-domain signal, which provides better recovery of the modulating signal properties acting at the sinoatrial node, is the HT signal. The PSD estimate that recovers the more accurate HRV spectrum estimates, also with ectopic beats, is the discrete Fourier transform of the HT signal.

\section{APPENDIX}

\section{Criterion to Detect Anomalous Beat Positions}

From the IPFM model point of view, the instantaneous heart rate is

$$
r(t)=\frac{1+m(t)}{T}
$$

and from the definition of $h t(t)$ in (3), $m^{\prime}(t)=h t^{\prime \prime}(t)$. With these relations the criterion of maximum heart rate variation $r^{\prime}(t)$ can be written as

$$
r^{\prime}(t)=\left|\frac{m^{\prime}(t)}{T}\right|=\left|\frac{h t^{\prime \prime}(t)}{T}\right|<U .
$$

Using three consecutive beats, $t_{k-1}, t_{k}$, and $t_{k+1}$, and the Lagrange's interpolation formula for nonuniformly distributed samples [24], $h t(t)$ can be estimated in the neighborhood of $t_{k}$ as

$$
\begin{aligned}
\widehat{h t}(t)= & h t\left(t_{k-1}\right) \frac{\left(t-t_{k}\right)\left(t-t_{k+1}\right)}{\left(t_{k-1}-t_{k}\right)\left(t_{k-1}-t_{k+1}\right)} \\
& +h t\left(t_{k}\right) \frac{\left(t-t_{k-1}\right)\left(t-t_{k+1}\right)}{\left(t_{k}-t_{k-1}\right)\left(t_{k}-t_{k+1}\right)} \\
& +h t\left(t_{k+1}\right) \frac{\left(t-t_{k-1}\right)\left(t-t_{k}\right)}{\left(t_{k+1}-t_{k-1}\right)\left(t_{k+1}-t_{k}\right)}
\end{aligned}
$$


therefore, being

$$
\begin{aligned}
\widehat{h t^{\prime \prime}}\left(t_{k}\right)= & \frac{2 h t\left(t_{k-1}\right)}{\left(t_{k-1}-t_{k}\right)\left(t_{k-1}-t_{k+1}\right)} \\
& +\frac{2 h t\left(t_{k}\right)}{\left(t_{k}-t_{k-1}\right)\left(t_{k}-t_{k+1}\right)} \\
& +\frac{2 h t\left(t_{k+1}\right)}{\left(t_{k+1}-t_{k-1}\right)\left(t_{k+1}-t_{k}\right)} .
\end{aligned}
$$

From the known beat positions, $t_{k}, h t(t)$ can be calculated at $t_{k-1}, t_{k}$, and $t_{k+1}$ following (5) as

$$
\begin{aligned}
h t\left(t_{k-1}\right) & =(k-1) T-k_{t_{k-1}} \\
h t\left(t_{k}\right) & =k T-t_{k} \\
h t\left(t_{k+1}\right) & =(k+1) T-t_{k+1}
\end{aligned}
$$

and then

$$
\widehat{h t^{\prime \prime}}\left(t_{k}\right)=2 T \frac{t_{k-1}-2 t_{k}+t_{k+1}}{\left(t_{k-1}-t_{k}\right)\left(t_{k-1}-t_{k+1}\right)\left(t_{k}-t_{k+1}\right)} .
$$

Thus, (15) can be estimated as

$$
\widehat{r^{\prime}}\left(t_{k}\right)=2\left|\frac{t_{k-1}-2 t_{k}+t_{k+1}}{\left(t_{k-1}-t_{k}\right)\left(t_{k-1}-t_{k+1}\right)\left(t_{k}-t_{k+1}\right)}\right|<U .
$$

\section{REFERENCES}

[1] M. Malik and A. J. Camm, Heart Rate Variability. New York: Futura, 1995.

[2] M. Malik, A. Xia, J. Poloniecki, O. Odemuyiwa, T. Farrell, A. Staunton, and A. J. Camm, "Influence of the noise and artefact in automatically analyzed long term electrocardiograms on different methods for timedomain measurement of heart rate variability," Comput. Cardiol., pp. 269-272, 1992.

[3] C. L. Birkett, M. G. Kienzle, and G. A. Myers, "Interpolation over ectopic beats increases low frequency power in heart rate variability spectra," Comput. Cardiol., pp. 257-259, 1992.

[4] N. Lippman, K. M. Stein, and B. B. Lerman, "Comparison of methods for removal of ectopy in measurement of heart rate variability," Amer. J. Physiol., vol. 267, pp. H411-418, 1994.

[5] P. Albrecht and R. J. Cohen, "Estimation of heart rate power spectrum bands from real-world data: dealing with ectopic beats and noisy data," Comput. Cardiol., pp. 311-314, 1989.

[6] R. M. Berne and M. Levy, Cardiovascular Physiology, 6th ed. St. Louis, MO: Mosby Year Book, Inc., 1992.

[7] M. Brennan, M. Palaniswami, and P. Kamen, "A new model-based ectopic beat correction algorithm for heart rate variability," presented at the 23rd Annu. Conf. IEEE/EMBS, Istanbul, Turkey, 2001.

[8] J. Mateo and P. Laguna, "Improved heart rate variability time-domain signal construction from the beat occurrence times according to the IPFM model," IEEE Trans. Biomed. Eng., vol. 47, pp. 985-996, Aug. 2000 .

[9] M. Malik, T. Farrell, T. Cripps, and A. Camm, "Heart rate variability in relation to prognosis after myocardial infarction: selection of optimal processing techniques," Eur. Heart J., vol. 10, pp. 1060-1074, 1989.

[10] D. Sapoznikov, M. H. Luria, Y. Mahler, and M. S. Gotsman, "Methods of arrhythmia removal in heart rate variability analysis," IEEE Eng. Med. Biol. Mag., vol. 13, pp. 668-669, 1991

[11] D. Sapoznikov, M. H. Luria, and M. S. Gotsman, "Comparison of different methodologies of heart rate variability analysis," Comput. Meth. Programs Biomed., vol. 41, pp. 69-75, 1993.

[12] A. Taddei, G. Distante, M. Emdin, P. Pisani, G. B. Moody, C. Zeelenberg, and C. Marchesi, "The European ST-T database: standards for evaluating systems for the analysis of ST-T changes in ambulatory electrocardigraphy," Eur. Heart J., vol. 13, pp. 1164-1172, 1992.

[13] E. J. Bayly, "Spectral analysis of pulse frequency modulation in the nervous systems," IEEE Trans. Biomed. Eng., vol. BME-15, pp. 257-265, Oct. 1968
[14] O. Rompelman, J. B. Snijders, and C. van Spronsen, "The measurement of heart rate variability spectra with the help of a personal computer," IEEE Trans. Biomed. Eng., vol. BME-29, pp. 503-510, July 1982.

[15] O. Rompelman, A. Coenen, and R. Kitney, "Measurement of heart-rate variability: Part 1 -comparative study of heart-rate variability analysis methods," Med. Biol. Eng. Comput., vol. 15, pp. 239-252, 1977.

[16] R. W. DeBoer, J. M. Karemaker, and J. Strackee, "Spectrum of a series of point event, generated by the integral pulse frequency modulation model," Med. Biol. Eng. Comput., vol. 23, pp. 138-142, 1985.

[17] — - "Comparing spectra of a series of point events particularly for heart rate variability data," IEEE Trans. Biomed. Eng., vol. BME-31, pp. 384-387, Apr. 1984.

[18] R. D. Berger, S. Akselrod, D. Gordon, and R. J. Cohen, "An efficient algorithm for spectral analysis of heart rate variability," IEEE Trans. Biomed. Eng., vol. BME-33, pp. 900-904, Sept. 1986.

[19] P. Castiglioni, "Evaluation of heart rhythm variability by heart or heart period: differences, pitfalls and help from logarithms," Med. Biol. Eng. Comput., vol. 33, pp. 323-330, 1995.

[20] N. R. Lomb, "Least-squares frequency analysis of unequaly spaced data," Astrophys. Space Sci., vol. 39, pp. 447-462, 1976.

[21] P. Laguna, G. B. Moody, and R. G. Mark, "Power spectral density of unevenly sampled data by least-square analysis: performance and application to heart rate signals," IEEE Trans. Biomed. Eng., vol. 45, pp. 698-715, June 1998.

[22] ESC/NASPE Task Force, "Heart rate variability: standards of measurement, phisiological interpretation, and clinical use," Ann. Noninvasive Electrocardiol., vol. 1, pp. 151-181, Apr. 1996.

[23] J. García, P. Lander, L. Sörnmo, S. Olmos, G. Wagner, and P. Laguna, "Comparative study of local and Karhunen-Loève based ST-T indexes in recordings from human subjects with induced myocardial ischemia," Comput. Biomed. Res., vol. 31, pp. 271-292, Aug. 1998.

[24] B. P. Demidovich and I. A. Maron, Computational Mathematics. Moscow, Russia: MIR, 1981.

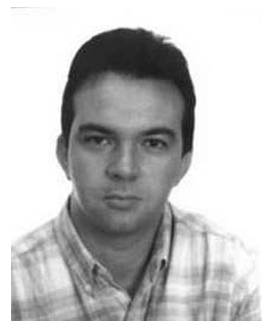

Javier Mateo was born in Zaragoza, Spain, in 1964. He received the M.Sc. degree in electrical engineering from the Polytechnic University of Madrid (U.P.M.), Madrid, Spain, and the Ph.D. degree from the University of Zaragoza (U.Z.), Zaragoza, Spain, in 1989 and 2000, respectively.

From 1989 to 1993, he was with Cables de Comunicaciones S. A., Zaragoza, Spain, where he worked on fiber optic sensors and optical communications. In 1993, he joined the Electronic Engineering and Communications Department of the University of Zaragoza, where he developed his Ph.D. degree dissertation. He is currently Assistant Professor of Optical Fiber Communications in the Department of Electronic Engineering and Communications at the Polytechnic Centre of the University of Zaragoza. His professional research interests are in signal processing, in particular, applied to biomedical signals, fiber optic sensors, and optical communication systems.

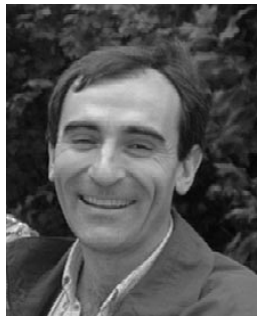

Pablo Laguna (M'92) was born in Jaca (Huesca), Spain, in 1962. He received the M.S. degree in physics and the Ph.D. degree from the University of Zaragoza (U.Z.), Zaragoza, Spain, in 1985 and 1990, respectively. The Ph.D. degree dissertation was developed at the Biomedical Engineering Division of the Institute of Cybernetics (I.C.), Polytechnic University of Catalonia (U.P.C.)-C.S.I.C., Barcelona, Spain.

He is currently an Associate Professor of Signal Processing and Communications in the Department of Electronic Engineering and Communications at the Centro Politécnico Superior, University of Zaragoza. From 1987 to 1992, he worked as Assistant Professor in the Department of Control Engineering at the Polytechnic University of Catalonia, Barcelona, Spain, and as a Researcher at the Biomedical Engineering Division of the Institute of Cybernetics. His professional research interests are in signal processing, in particular, applied to biomedical applications. 